\title{
The use of hypertext and sensory-level supports for visual learning of aircraft names and shapes
}

\author{
JOSEPH PSOTKA \\ U.S. Army Research Institute \\ and \\ STEVE KERST and TRISH WESTERMAN \\ Catholic University of America and U.S. Army Research Institute
}

\begin{abstract}
Hypertexts have many novel and unexplored features for representing, linking, and exploring knowledge to make it more useful for learning. We contrasted traditional textbook presentation of information against a hypertext that implemented database retrieval of text and manipulation of colored, bitmapped displays. Hypertext enabled us to explore the role of sensory-level supports, such as apparent motion and colored overlays, as aids for visual cognition and concept formation. One visible difference between traditional text and hypertext was in the use of a rapid browsing strategy by the hypertext learners, compared with a methodical, extended side-by-side comparison by the book learners. The research also discovered that students better learned local neighborhoods of concepts, as opposed to widely dispersed concepts, in the hypertext environment. We selected visual aircraft recognition (VACR) as a topical area for the research because it is a very important problem in the Army, because traditional VACR training emphasizes both text and images, and because we had available to us a fine manual (Army Field Manual FM 44-30) full of black-and-white pictures and text that was artfully arranged on the page, as well as access to hundreds of color slides of airplanes. Our research found superior learning with the hypertext field manual, and we examined higher order learning strategies, such as depth-first versus breadthfirst paths through the hierarchy of knowledge, and sensory-level supports for learning within these environments.
\end{abstract}

It is the considerable convenience that hypertext has added to mixing pictures and text for rapid browsing (Mandl \& Levin, 1989; Psotka, Massey, \& Mutter, 1988) that motivates this research on visual learning. The main goal of this research was to determine how low-level sensory processes in multimedia environments can contribute to the two basic elements of learning: discrimination and categorization. In discrimination, differences among visual objects must be recognized and stored; in classification, or categorization, smaller differences must be overlooked to determine the essential similarity among visual objects (Tennyson \& Park, 1980). Discrimination must essentially decompose images and objects into their constituent features. Categorization must determine what constellation of features is important enough to use as a base for clustering objects. Animation in particular has been a difficult manipulation to apply to learning (Freeman, 1924; Park \& Gittelman, 1992), and thus it has

\footnotetext{
The opinions in this paper do not necessarily imply or express the views of the U.S. Army Research Institute (USARI). We thank Melissa Holland, Jonathan Kaplan, John Pliler, Michelle Sams, Kirk Thor, and the many Army developers of FM 44-30 for their help. This research was funded by the USARI Basic Research Office. Correspondence should be addressed to J. Psotka at the U.S. Army Research Institute (Attention: PERI-ICC), 5001 Eisenhower Ave., Alexandria, VA 22333-5600 (e-mail address: psotka@alexandria-emh2.army.mil).
}

had a checkered effect. Computer multimedia technology may offer a new understanding of its potential role.

Another goal of this research was to devise and assess novel techniques, such as central nodes of "browsers," that can act as "launch platforms" from which to investigate the structure of multimedia databases. Improper use of the power of hypertext can result in chaos and confusion, a syndrome widely known as being "lost in hyperspace." Uncovering what sort of learning strategies people can adopt to overcome the complexities and chaos of hyperspace for visual learning was the fundamental goal of this research effort.

We constructed a visual hypermedia database by digitizing Army Field Manual FM 44-30 with many novel features afforded by point-and-click technology. We tried to maintain the integrity of the original book, only adding new search and comparison facilities rather than explicitly reorganizing the materials. The layout of the hypertext page is shown in Figure 1.

\section{Sensory-Level Aids to Visual Comparison}

1. Side-by-side contrast. Side-by-side viewing of two similar airplanes by clicking on the name of the similar airplane in the text field labeled "similars."

2. Color contrast. Superimposed images of the outline of one airplane over a similar plane in a three-color meld, 


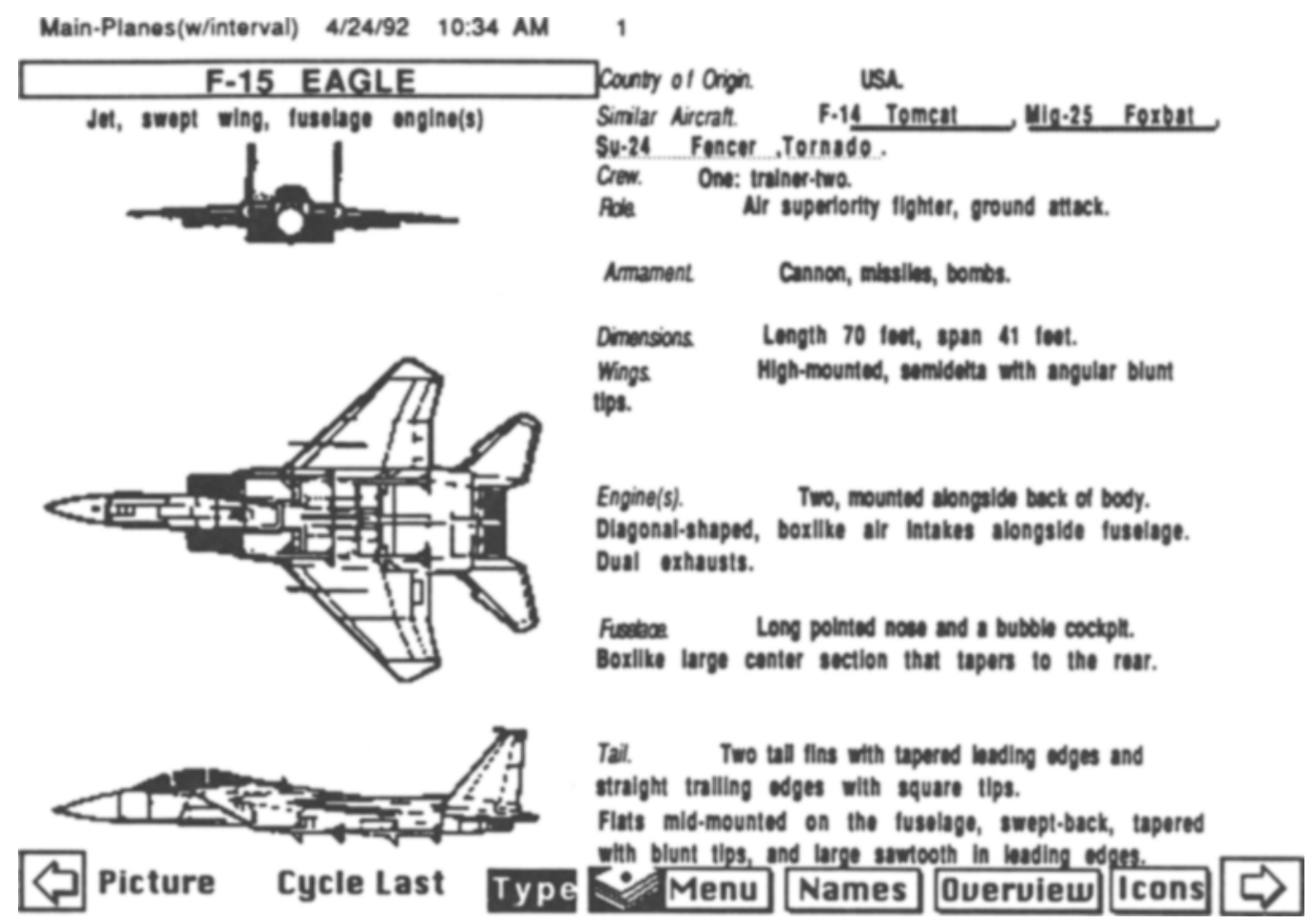

Figure 1. Physical layout of the hypertext page.

in which one plane is red, the other green, and the overlap yellow.

3. Apparent motion contrast. Alternate presentations of $.6 \mathrm{sec}$ each, with a variable interval between two images' presentation, of one airplane, then a similar one, in rapid succession at the same location on the screen. The images of all similar airplanes (those airplanes in the "Similar" list on a page) had been carefully scaled and oriented to be in registry with each other on a page.

4. Airplane browser. A visual space was constructed on the basis of clustering similar aircraft. This Airplane Browser could be used as a launch platform to visit all of the planes, and the similar planes surrounding each plane, by clicking on the icon of each plane.

5. Picture. Photographs of each plane were digitized and made available by clicking on the "Picture" button on each card. This generated a 70-dpi bitmap on the screen, with 256 gray levels.

\section{EXPERIMENT 1}

Experiment 1 was an attempt to find out how easy this complicated system was to use. Since we had developed the hypertext, and had tried to make it as efficient and pleasant to use as possible, we wanted to get user reactions to the environment and compare them with its bookversion source. All of the features that we added seemed to have some merit to us for learning the planes, but we could not easily foretell which features people would actually use. In keeping with the purpose of possibly using this as a replacement for the Army Field Manual, we decided to compare how well people could use the hypertext to learn the names of a subset of airplanes.

\section{Method}

\section{Subjects}

Ten students ( 5 men and 5 women) were drawn from the local university undergraduate population. They were each paid $\$ 10$ to participate in the experiment.

\section{Stimuli}

Two experimental groups were used. Each student was given either the FM 44-30 manual (book group) or its hypertext version (hypertext group) written in HyperCard on a Macintosh IIci. Both groups were given the pretest and posttest on the Macintosh IIci.

Twenty planes were selected to sample the space of all 90 planes in the hypertext. For each plane, three names were selected as distractors in the pre- and posttest such that at least one distractor was a similar plane (i.e., on the "Similar" list for the plane) or came from the same category of planes (fighter, bomber, cargo plane, etc.).

\section{Procedure}

The subjects were instructed on the general goals of the experiment, were given a pretest on how well they could name each of 20 airplanes, and then were allowed to pursue their own strategies in learning the names of 20 planes during 30 min of study. They were then given a posttest identical to the pretest.

\section{Results}

The subjects were able to learn the names of most of the planes in both conditions. There were no pretest percent correct recognition differences. However, the hyper- 
text group outperformed the book group on the posttest [ 17 vs. 12.8 planes correctly identified on the posttest; $t(8)=3.77, p<.01]$.

\section{Student Comments}

On the whole, the subjects were more favorably disposed to the hypertext than to the book. Four of the 5 book group subjects felt that they needed more time, whereas none of the hypertext group made that comment. Two of the book group subjects said that they had a hard time finding the planes, whereas none of the hypertext group said that. On the other hand, 1 of the hypertext group subjects said that there were too many pieces of information in the hypertext and that a book version would be easier; however, 2 subjects said that the hypertext system was interesting and easy to learn to use.

\section{Discussion}

We showed that student novices could use hypertext more easily than they could use a book to learn the names of airplanes, even though they were more familiar with the medium of a book. The next step was to analyze how they used these new materials. Although we could easily force students to use the many new features by instructing them to do so, we decided to explore more indirect methods to persuade them to use the comparison and contrast technologies embedded in the hypertext. By using these indirect techniques, we hoped to be able to analyze the ecological validity of each technique more conclusively. Besides, it was not at all clear to us which of these techniques would prove superior, and in what mix. Since there are so many options available for learning in this hypertext, it seemed unlikely that any one option would be best for everyone or should be used to the exclusion of all other options. It seemed most sensible to encourage students to use them all and for us to try to discover clues from their responses about which techniques were most effective, and under what conditions.

\section{EXPERIMENT 2}

It is relatively easy to program a computer to record all of the actions that a user takes. Experimenters must be selective in their data requests, or they will be inundated with trivial data. Even then, it takes a thorough understanding of the environment to make sense out of the myriad mouse clicks and keystrokes that may be recorded. This is particularly true in a hypertext in which users are free to direct themselves through a complex space along many different paths. Learners are free to set their own goals and determine their own plans for achieving those goals. Discerning from a few mouse clicks what those goals might be is a challenging problem for experimenters.

We decided to collect mouse clicks only for the main comparison events we had built into the hypertext. We collected information on when a card was opened and closed, when side-by-side similar planes were viewed, when a picture was opened, when a color contrast was viewed, when an apparent motion flash was used, and when the PlaneSpace browser was used. Each of these occurrences were time stamped and recorded in a subject file using HyperTalk scripts.

\section{Method}

\section{Subjects}

Fifteen students ( 8 men and 7 women) were drawn from the local university undergraduate population. They were each paid $\$ 20$ to participate in this experiment.

\section{Stimuli}

Instructional medium. Two groups were tested, one using the book and the other the hypertext. Each student was assigned to one of the two instructional medium groups and given either the FM 4430 manual (book group) or its hypertext version (hypertext group) written in HyperCard on a Macintosh IIci. The pretest and posttest were given on the Macintosh IIci for both groups. The subjects in the book group were instructed to make side-by-side comparisons by locating the two planes in the book and then folding over the intervening pages to expose the two planes side by side. They were explicitly taught this strategy so that we might be able to determine the added value of our contrast techniques.

Plane organization. Two levels of plane organization were used: pairs and cluster. Eight pairs of planes were selected to sample the space of all 90 planes in the hypertext. In addition, one small cluster of 2 pairs that were all similar to each other was used.

For each plane, three names were selected as distractors in the pre-and posttest such that at least one distractor was a similar plane (i.e., on the "Similar" list for the plane) or came from the same category of planes (fighter, bomber, cargo plane, etc.). On the preand posttest, these pairs of planes were shown side by side, but the list of four alternative names included the name of only one of the planes. Each paired comparison was tested twice with slightly different views of the outline drawings showing only two of the three views (bottom, side, and front) at a time.

Transfer test. In addition to these tests, a surprise transfer test was given, using photographs of each of the 20 planes. These were new photographs that had not been previously shown to the learner. The learner had to name the plane shown on the photograph. It was our hope that this transfer test would reveal more enduring side effects of the study period.

\section{Results}

\section{Pretest}

All students successfully completed the experiment. There were no pretest percent correct recognition differences (13.8 for the hypertext group vs. 14.6 for the book group).

\section{Posttest}

A two-factor analysis of variance was calculated using repeated measures on each of the 20 planes, crossing performance in the two instructional medium groups with the two plane organizations: one small cluster of 2 pairs against all of the other 8 pairs of planes (see Figure 2). The posttest percent correct recognition showed a significant difference $[F(1,18)=4.5, p<05 ; M=56.7$ for the hypertext group vs. $M=48.7$ for the book group]. The main effect of plane organization (cluster vs. pairs) was not significant. The interaction of Plane Organization with instructional medium was significant $[F(1,18)=$ $11.1, p<.01]$ 


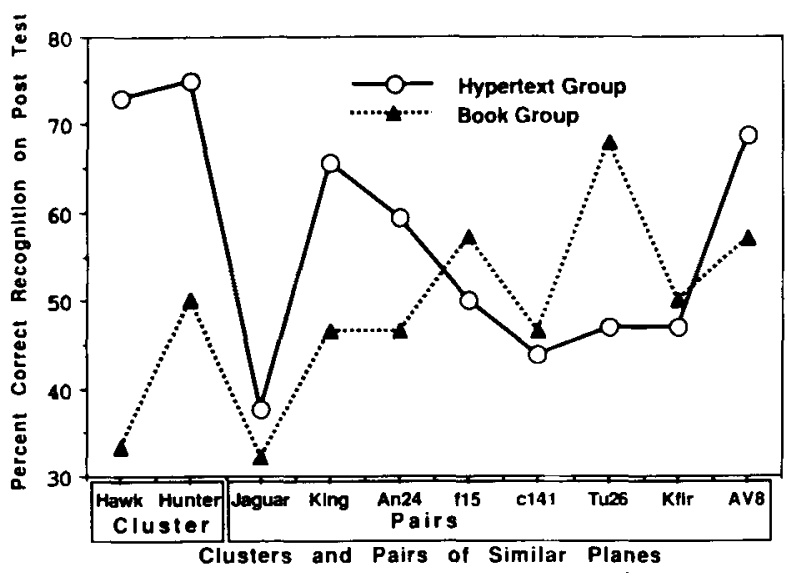

Figure 2. Percent correct recognition on the posttest as a function of instructional medium and plane organization.

\section{Transfer Test}

The transfer test using new photographs yielded a main effect for instructional medium that was significant using a paired $t$-test on performance on each of the 20 planes [41.6 for the hypertext group vs. 30.0 for the book group; $t(18)=2.81, p<.01]$. The main effects for plane organization and the interaction of plane organization with instructional medium were not significant.

\section{Individual Analysis}

The hypertext group made extensive use of all the comparison and contrast features we built into the system. On the average, each subject used at least one of the comparison techniques (color contrast, side-by-side comparison, or apparent motion contrast) with each plane pair. In addition, 1 student used the PlaneSpace browser extensively. Correlations were carried out between these measures and the post- and transfer tests. There were no significant correlations with the posttest results (the transfer test correlations were described above).

\section{Rapid Browsing}

The most striking result replicated the first experiment and provided quantitative detail about the overarching strategy that all students adopted, that of rapid browsing. The students in the book group visited each plane only 2.3 times, whereas the students in the hypertext group visited each plane an average of 5.2 times. Figure $3 \mathrm{dem}-$ onstrates another noticeable feature: The variance in the number of looks at each plane shows how the subjects individualized their study time. The five highest frequencies are for these five planes: Jaguar, Hunter, Yak, AV8, and Hawk. Four of these planes are in the cluster group. Presumably, the subjects spent more time on the difficult planes and made more efficient allocation of their limited time.

Analysis of the browsing habits of these students revealed a common feature: They tended very frequently to study similar planes in sequence. That is, they would examine a plane, compare and contrast it with a similar plane using colored contrast, animated flash, or side-byside comparison, and then they would go to the card for that similar plane and study the same pair.

\section{Side-by-Side Similars}

The comparison of the subjects in the book and hypertext groups on the frequency with which they used side-by-side comparison of similar planes needs some explanation. The book group could make side-by-side comparisons by locating the two planes in the book and then folding over the intervening pages to expose the two planes side by side. They were explicitly taught this strategy so that we might be able to determine the added value of our contrast techniques. It turns out that both groups used side-by-side comparisons roughly equally. The significant correlation between the book and hypercard groups is $.53(18 d f, p<.05)$.

\section{Discussion}

The analysis of this experiment suggests that hypertext provides some superior supports for learning beyond the basic access superiority found in Experiment 1. These advantages for learning include strategic and sensory-level components. To summarize, the advantages of hypertext include:

1. Rapid access to individual cards or data objects. This resulted in a strategic decision by many subjects to rapidly browse through many cards many times (four to six times), rather than just twice, as is normally done with a book.

2. Superior memory for a group of four or more individual objects when they are all part of the same cluster of very similar objects. This finding agrees cleanly with our theoretical conceptions that hypertext comparisons are particularly useful when there are many links or connections among nodes that must all be traversed in short order. The strength of hypertext is that these linkages are readily available to help lower-level sensory analyses support cognitive processing.

3. Superior transfer of these recognition skills to novel instances.

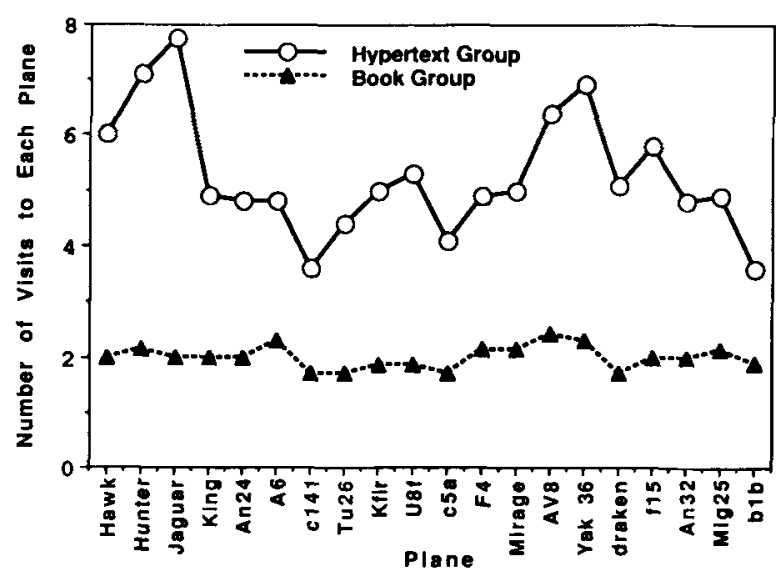

Figure 3. Variance in the number of looks at each plane as a function of instructional medium and plane organization. 


\section{Sequential Similars}

One common feature that was uncovered by the individual analyses of the hypertext group was the high frequency of visiting similar planes in sequence. This tactic was second in frequency to side-by-side viewing. Why this should be so was not immediately apparent. However, we might speculate that this sequential viewing could be beneficial for comparison purposes. It might allow students to use a kind of extended apparent motion to contrast the similar planes, since it is only through sequential viewing that the planes are physically brought into registry by their location in space, not by eye movements as in side-by-side viewing. It may also be an instance of the "cut hypothesis" (Avrahami \& Kareev, 1992), in which a sequence of stimuli is cut out to become a cognitive entity if it is repeatedly experienced in different contexts.

The generally beneficial effects of rapid browsing and apparent motion have not been the focus of much experimental scrutiny for education and training. These findings of cognitive benefits agree with the apparent motion findings of Gregory (1991). He discovered that more rapid motion with apparent movement jumps of greater than about $10 \mathrm{~min}$ of arc of visual angle is analyzed by a cortical system that is different from the one that analyzes slower real or apparent motion. When the gaps in space or time are too large, the perception of motion is determined by more cognitive characteristics of the stimulus, such as whether the affected features are part of the same object (and thus likely to move together) or parts of different objects. The use of real or apparent motion to decompose objects into their constituent features is well known (cf. Proffitt \& Kaiser, 1991; Shebilske, 1991), but its use for learning, especially in broad domains of education such as science, math, and the humanities, remains unexplored.

\section{Testing on Computer}

One source of bias in previous experiments is that the tests were conducted on computers. The significant transfer test in Experiment 2 (not presented on the computer) was deliberately used to counter the argument that the subjects in the hypertext group had more experience with a computer, and that the training and testing materials were more alike, resulting in their superior performance. Superior performance on the transfer test belies this interpretation.

\section{General Implications for Education}

These results point to a manipulation of sensory-level interactions that support learning in ways that have either never before been possible or that are now on an order of magnitude easier than ever before. Theories that guide the use of these sensory-level supports for learning in principled ways may extend propaedeutic techniques significantly.

\section{REFERENCES}

Avrahami, J., \& Kareev, Y. (1992). Decomposition of temporal sequences. Proceedings of the Fourteenth Annual Cognitive Science Society (pp. 426-431). Bloomington: Indiana University Press.

FreEman, F. N. (1924). Visual education: A comparative study of motion pictures and other methods of instruction. Chicago: University of Chicago Press.

Gregory, R. L. (1991). Seeing by exploring. In S. R. Ellis, M. K. Kaiser, \& A. C. Grunwald (Eds.) Pictorial communication in virtual and real environments (pp. 47-60). London: Taylor \& Francis.

MANDL, H., \& LEVIN, J. R. (1989). Knowledge acquisition from text and pictures. New York: North-Holland.

Park, O. K., \& GitTelman, S. S. (1992). Computer-based animation and feedback in electronic troubleshooting skill acquisition. Educational Technology Research \& Development, 40(4), 27-38.

Proffit, D. R., \& Kaiser, M. K. (1991). Perceiving environmental properties from motion information: Minimal conditions. In $\mathbf{S}$. $\mathbf{R}$. Ellis, M. K. Kaiser, \& A. C. Grunwald (Eds.), Pictorial communication in virtual and real environments (pp. 47-60). London: Taylor \& Francis.

Psotka, J., Massey, L. D., \& Mutter, S. A. (1988). Knowledge representation. In J. Psotka, L. D. Massey, and S. A. Mutter (Eds.), Intelligent tutoring systems: Lessons learned ( $\mathrm{pp} .15-20$ ). Hillsdale, NJ: Erlbaum.

ShebiLSKE, W. (1991). Visuomotor modularity, ontogeny, and training high-performance skills with spatial instruments. In S. R. Ellis, M. K. Kaiser, \& A. C. Grunwald (Eds.), Pictorial communication in virtual and real environments (pp. 305-315). London: Taylor \& Francis, Ltd.

Tennyson, R. D., \& Park, O. K. (1980). The teaching of concepts: A review of instructional design research literature. Review of Educational Research, 50(1), 55-70. 